\title{
THE DETECTION RATE OF EARLY UV EMISSION FROM SUPERNOVAE: A DEDICATED GALEX/PTF SURVEY AND CALIBRATED THEORETICAL ESTIMATES
}

\author{
Noam Ganot ${ }^{1}$, Avishay Gal-Yam ${ }^{1}$, Eran. O. OfeK ${ }^{1}$, Ilan Sagiv $^{1}$, Eli Waxman ${ }^{1}$, Ofer Lapid ${ }^{1}$, Shrinivas R. Kulkarni ${ }^{2}$, \\ SAGi Ben-Ami ${ }^{3}$, Mansi M. Kasliwal ${ }^{2}$ \\ (The ULTRASAT SCIENCE TEAM), \\ Doron Chelouche ${ }^{4}$, StePhen Rafter $^{4}$, Ehud Behar $^{5}$, Ari LaOr ${ }^{5}$, Dovi Poznanski ${ }^{6}$, Ehud NaKar ${ }^{6}$, Dan MaOz $^{6}$, \\ BenNy TRAKHTENBRoT ${ }^{7,14}$ \\ (THE WTTH CONSORTIUM), \\ James D. Neill $^{8}$, Thomas A. Barlow ${ }^{8}$, Christofer D. Martin ${ }^{8}$, Suvi Gezari ${ }^{9}$ \\ (THE GALEX SCIENCE TEAM), \\ AND \\ Iair Arcavi ${ }^{10,15}$, Joshua S. Bloom ${ }^{11}$, Peter E. Nugent ${ }^{12}$, and Mark Sullivan ${ }^{13}$ \\ (The Palomar Transient Factory) \\ ${ }^{1}$ Department of Particle Physics and Astrophysics, Faculty of Physics, The Weizmann Institute of Science, Rehovot 76100, Israel; noam.ganot@gmail.com \\ ${ }^{2}$ Cahill Center for Astrophysics, California Institute of Technology, Pasadena, CA 91125, USA \\ ${ }^{3}$ Smithsonian Astrophysical Observatory, Harvard-Smithsonian Ctr. for Astrophysics, 60 Garden Street, Cambridge, MA 02138, USA \\ 4 Physics Department, Faculty of Natural Sciences, University of Haifa, 31905 Haifa, Israel \\ ${ }^{5}$ Physics Department, Technion Israel Institute of Technology, 32000 Haifa, Israel \\ ${ }^{6}$ School of Physics and Astronomy, Tel Aviv University, 69978 Tel Aviv, Israel \\ ${ }^{7}$ Institute for Astronomy, ETH Zurich, Wolfgang-Pauli-Strasse 27 Zurich 8093, Switzerland \\ ${ }^{8}$ California Institute of Technology, 1200 East California Boulevard, MC 278-17, Pasadena, CA 91125, USA \\ 9 Department of Astronomy, University of Maryland, College Park, MD 20742-2421, USA \\ ${ }^{10}$ Las Cumbres Observatory Global Telescope, 6740 Cortona Drive, Suite 102, Goleta, CA 93111, USA \\ 11 Department of Astronomy, University of California, Berkeley, CA 94720, USA \\ 12 Lawrence Berkeley National Laboratory, 1 Cyclotron Road, Berkeley, CA 94720, USA \\ ${ }^{13}$ School of Physics and Astronomy, University of Southampton, Southampton SO17 1BJ, UK \\ Received 2015 December 19; accepted 2016 February 4; published 2016 March 17
}

\begin{abstract}
The radius and surface composition of an exploding massive star, as well as the explosion energy per unit mass, can be measured using early UV observations of core-collapse supernovae (SNe). We present the first results from a simultaneous GALEX/PTF search for early ultraviolet (UV) emission from SNe. Six SNe II and one Type II superluminous SN (SLSN-II) are clearly detected in the GALEX near-UV (NUV) data. We compare our detection rate with theoretical estimates based on early, shock-cooling UV light curves calculated from models that fit existing Swift and GALEX observations well, combined with volumetric SN rates. We find that our observations are in good agreement with calculated rates assuming that red supergiants (RSGs) explode with fiducial radii of $500 R_{\odot}$, explosion energies of $10^{51} \mathrm{erg}$, and ejecta masses of $10 M_{\odot}$. Exploding blue supergiants and Wolf-Rayet stars are poorly constrained. We describe how such observations can be used to derive the progenitor radius, surface composition, and explosion energy per unit mass of such SN events, and we demonstrate why UV observations are critical for such measurements. We use the fiducial RSG parameters to estimate the detection rate of SNe during the shock-cooling phase ( $<1$ day after explosion) for several ground-based surveys (PTF, ZTF, and LSST). We show that the proposed wide-field UV explorer ULTRASAT mission is expected to find $>85 \mathrm{SNe}$ per year $\left(\sim 0.5 \mathrm{SN}\right.$ per $\left.\mathrm{deg}^{2}\right)$, independent of host galaxy extinction, down to an NUV detection limit of $21.5 \mathrm{mag} \mathrm{AB}$. Our pilot GALEX/PTF project thus convincingly demonstrates that a dedicated, systematic SN survey at the NUV band is a compelling method to study how massive stars end their life.
\end{abstract}

Key words: supernovae: general

Supporting material: animation

\section{INTRODUCTION}

Massive stars explosively end their life as a Core Collapse Supernova (CC SN), but few solid facts are known about SN progenitors. Hydrogen-rich SNe II (and in particular, Type II-P) are firmly associated with red supergiant (RSG) progenitors, while rare underluminous SNe II (e.g., SN 1987A) may arise from blue superiants (BSG). Other classes of core-collapse $\mathrm{SNe}$

\footnotetext{
${ }_{15}$ Zwicky Fellow.

${ }^{15}$ Kavli Institute for Theoretical Physics, University of California, Santa Barbara, CA 93106, USA.
}

that are depleted in hydrogen (e.g., Types Ib, Ic) probably arise from stripped stars, such as Wolf-Rayet (W-R) stars, but the exact mapping is unknown (see Filippenko 1997 and Smartt 2009 for reviews of SN types and progenitors, respectively). The final stages of massive star evolution and the physics of the explosion are also poorly understood (see, e.g., Langer 2012 and references therein).

Although there are numerous SN detections every year (GalYam et al. 2013), most events are discovered a long time (days) after the explosion of the star. This delay is unfortunate because radiation emitted during the first few days after $\mathrm{SN}$ explosion is 
governed by relatively simple physics: recombination and line opacity are negligible and in most cases so is radioactivity. This early emission encodes crucial information about the outer envelope of the exploding star (approximately its outer $\left.0.1 M_{\odot}\right)$, which can be extracted from robust and simple models. Exploring this outer shell mass is very interesting because it is what determines the stellar radius and outer density profile of the star, and its properties can be used to study currently poorly known stellar physics, such as the mixing length and convection parameters. Observations starting only after this early period thus result in loss of this information about the $\mathrm{SN}$ progenitor star and the explosion mechanism itself. Only a handful of events were detected during this early phase (e.g., Arnett et al. 1989; Schmidt et al. 1993; Campana et al. 2006; Gezari et al. 2008, 2010; Schawinski et al. 2008; Soderberg et al. 2008; Arcavi et al. 2011; Gal-Yam et al. 2011, 2014; Cao et al. 2013), and even in these cases the time resolution of the measurements is generally too poor to form a well-sampled light curve.

An early detection of the SN and a measurement of its light curve are useful to understand the physics of the explosion itself, as well as its progenitor properties. The first light escaping from an exploding star emerges as a shock-breakout flare, with a hot spectrum peaking in the ultraviolet (UV) or $\mathrm{X}$-ray bands. Models for this shock-breakout emission have a long history (e.g., Grassberg et al. 1971; Colgate 1974; Falk 1978; Klein \& Chevalier 1978; Ensman \& Burrows 1992; Matzner \& McKee 1999). In recent years several theoretical models were developed to describe emerging observations of the explosion shock breakout (e.g., Nakar \& Sari 2010; Sapir et al. 2011, 2013; Katz et al. 2012). Figure 1 shows that exact analytic and numerical solutions by Sapir et al. $\left(2013^{16}\right.$; hereafter SKW13) are in general agreement with analytic models by Nakar \& Sari (2010; hereafter NS10) after appropriate rescaling of the latter. ${ }^{17}$ However, only a single such flare has been serendipitously observed (Soderberg et al. 2008) and the relevant theory is virtually untested. If detected, shock-breakout flares provide a direct measure of the pre-explosion stellar radius $R_{*}$ : the flare duration scales as $R_{*} / c$, and the integrated luminosity as $R_{*}^{2}$ (Klein \& Chevalier 1978; Katz et al. 2012).

Following an initial shock-breakout flare (i.e., at times $\gtrsim 3 \mathrm{hr}$ post explosion), the expanding stellar envelope emits a fraction of the leftover stored explosion energy during the shockcooling phase, initially peaking in the UV. This phase is better understood theoretically (e.g., Grassberg et al. 1971; Chevalier 1976, 1992; Chevalier \& Fransson 2008) and has been observed in a few cases (by GALEX, Gezari et al. 2008; Schawinski et al. 2008; and by Swift, Soderberg et al. 2008; data shown as red and black circles in Figure 1). The shockcooling phase is longer and more luminous in larger stars. These works and, in particular, more recent models (e.g., Nakar \& Sari 2010; Rabinak \& Waxman 2011, hereafter RW11)

\footnotetext{
16 The values in Table 2 of Sapir et al. (2013) were extended to include $\chi=0.01$ and 0.03 (N. Sapir 2016, personal communication). A modified blackbody spectrum is used to find the band luminosity rather than a Wien spectrum, as described in their paper, because the comptonized spectrum is similar in shape to a Wien spectrum only when the temperature is of the same order of the typical band energy. In our case (NUV) the temperature is $\sim 10$ times larger than the band energy and a modified blackbody spectrum better describes the comptonized spectrum for this band.

${ }^{17}$ To correct the NS10 formulae we divided the luminosity in their Equations (29), (32), and (39) by a factor of 2.5 (E. Nakar 2016, personal communication).
}

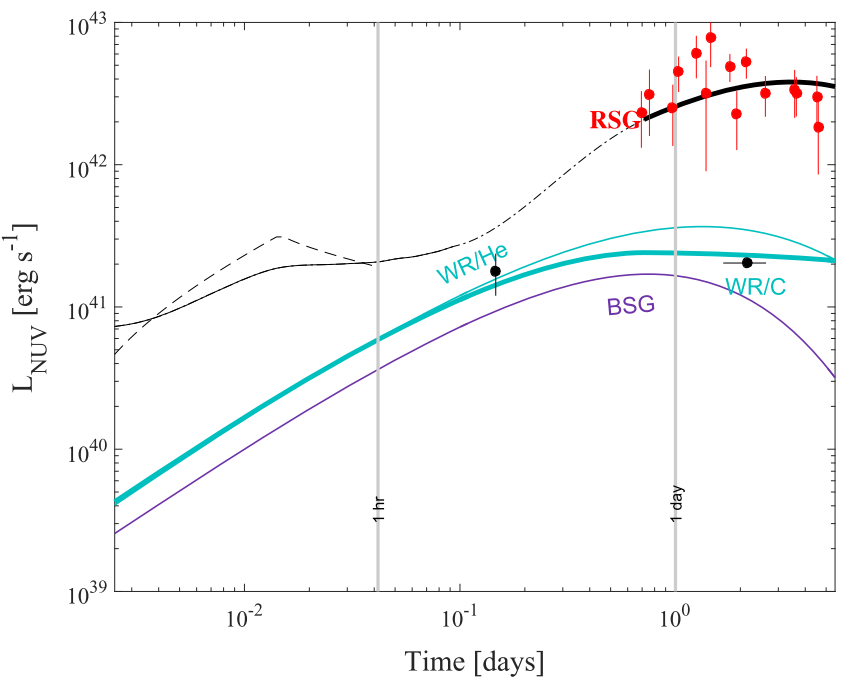

Figure 1. SN early NUV emission models compared to data. The RSG and BSG light curves are in the GALEX NUV band, while the WR light curves are in the SWIFT UVW1 band. At early times ( $<3 \mathrm{hr}$ post explosion) no optical/ UV data exist. The models of Sapir et al. (2013, thin solid) and Nakar \& Sari (2010, rescaled, see text; dashed) for these early times approximately agree on the peak value, but differ in details. The time axis for the SKW13 model was arbitrary shifted by $3 \times t_{0}$. (The zero time of the SKW13 model is defined as the time the shock arrives to the progenitor surface. At this time both the bolometric luminosity and the temperature already reached their peak. To plot the rise of the bolometric luminosity and temperature to their peak on a logarithmic timescale, the time axis should be shifted. A similar shift is used in SKW13, Figures 6-8. The shift size is three times the dynamic time of the model, $t_{0}$. This shift is negligible comparing to the timescale of RW11, allowing to interpolate between the two models.) Forthcoming UV surveys (e.g., ULTRASAT; Sagiv et al. 2014) will observe such early emission and further constrain models. At later times, Rabinak \& Waxman (2011) model for red supergiant stars (RSG; thick black) and compact W-R stars (He, thick cyan, and $\mathrm{C} / \mathrm{O}$, thin cyan) compare well with UV observations from Swift/UVOT (SN 2008D, Type Ib, Soderberg et al. 2008, black solid circles) and GALEX/ NUV (SNLS-04D2dc, Type II, Gezari et al. 2008; Schawinski et al. 2008, red solid circles). RW11 Blue supergiant (BSG) model (thin purple) are currently untested. Stellar classes (RSG/BSG/WR) differ greatly in their UV peaks making early UV observations a strong discriminator among progenitor classes. Plotted models assume the reasonable parameters for an RSG star with $R_{*}=500 R_{\odot}$, explosion energy $E=2 \times 10^{51} \mathrm{erg}$, and ejected mass $M=10$ $M_{\odot}$; for a BSG star with $R_{*}=50 R_{\odot}, E=10^{51} \mathrm{erg}$, and $\mathrm{M}=10 M_{\odot}$. The models for the W-R star, either He or $\mathrm{C} / \mathrm{O}$ dominated composition, and the RW11 fitted parameters for SN2008D with $R_{*}=10^{11} \mathrm{~cm}, E=6 \times 10^{51} \mathrm{erg}$, and ejected mass $M=7 M_{\odot}$. RW11 models are unextinguished, data points have been extinction corrected (by $A_{\mathrm{NUV}}=1.45 \mathrm{mag}$ and $A_{\mathrm{NUV}}=2.2 \mathrm{mag}$ for SNLS-04D2dc and SN 2008D, respectively) using the extinction values provided by Schawinski et al. (2008) and RW11 (for SN 2008D). Note that the parameters that appear in RW11 for SNLS-04D2dc were constrained by an uncommon star profile with $f_{\rho}=25$ (see explanation in RW11), in order to compare their model with the one used by Gezari et al. (2008). We demonstrate here that a common star profile with $f_{\rho}=0.1$ may also fit this data. The SKW13 model is valid up to $t_{\mathrm{sph}}$, while the RW11 model is valid starting from $t_{\mathrm{BO}}$ (see papers for the exact definitions of those times). A logarithmic spline interpolation (dash-dot) is plotted between the two models for RSGs.

demonstrate that the shock breakout and subsequent cooling phases during the first days after explosion encode information about the SN progenitor radius and surface composition, the explosion energy per unit mass, and the line of sight extinction (see the following for details). This is strong motivation to design surveys targeting early UV emission from SNe. The importance of UV measurements in early times was also raised by Pritchard et al. (2014). In this paper we use the results of a pilot PTF/GALEX survey to robustly estimate the number of early SN detections expected from such surveys. 
We review the derivation of physical progenitor and SN parameters from early observations of $\mathrm{SNe}$ in Section 2 and describe a sample of SNe with early UV emission detected by a GALEX/PTF wide-field experiment in Section 3. We summarize our implementation of theoretical models in Section 4 and show that these fit the handful of available data. We then combine these models with volumetric $\mathrm{SN}$ rates to estimate the expected number of detections from the GALEX experiment we conducted in Section 5, show our fiducial models fit the observations well, and provide validated predictions for the proposed ULTRASAT space mission (Sagiv et al. 2014). We conclude in Section 6.

\section{MOTIVATION: DERIVING SN PROGENITOR PROPERTIES FROM EARLY UV EMISSION}

The early shock-cooling emission from $\mathrm{SNe}$ is governed by simple and well understood physics and can thus be used to derive robust constraints on the physical parameters of the exploding star and of the explosion. Roughly, the rise time to peak determines the progenitor radius $R_{*}$, the peak flux determines the explosion energy per unit ejecta mass $E / M$, and the post-peak light curve constrains the surface composition $Z$ (RW11). This simple physics description holds as long as the temperature in the emitting region is $\gtrsim 1 \mathrm{eV}$ (see RW11 for details $^{18}$ ), for which the emission peak is at $\lambda<0.3 \mu \mathrm{m}$. In all optical bands (including the $u$ band) the emission peak occurs only after the temperature falls below this threshold value (see Rubin et al. 2015 for detailed discussion). For this reason, the observed photometric parameters (rise time to peak, peak flux) cannot be related to physical parameters via a simple and robust model, making optical light observations not useful for this analysis.

For commonly assumed progenitor parameters, shock breakout is expected to be accompanied by soft $(0.3-10 \mathrm{keV})$ $\mathrm{X}$-ray emission with a luminosity of $10^{45} \mathrm{erg} \mathrm{s}^{-1}\left(10^{44} \mathrm{erg} \mathrm{s}^{-1}\right)$ for BSG (RSG/He-WR) progenitors (Sapir et al. 2013). However, the ability to use X-ray observations to constrain progenitor and explosion parameters is limited by several factors.

i. First, the theory of X-ray emission from massive star explosions is not sufficiently well understood to ensure that stellar/explosion parameters can be reliably constrained based on X-ray observations. This is reflected, for example, by the fact that none of the few X-ray detections can be explained as shock breakout from a stellar edge (e.g., Sapir et al. 2013); instead, these require more complex structures (such as winds or extended envelopes, e.g., Campana et al. 2006; Moriya et al. 2015).

ii. Second, the detection rate of X-ray breakouts is expected to be very low, even for future instruments with order of magnitude better sensitivities than past or current instruments. The non-detection of the predicted

\footnotetext{
${ }^{18}$ Several complications that prevent the construction of a simple and robust model arise when the temperature of the emitting region drops below $\sim 1 \mathrm{eV}$ (RW11): complicated opacity variations, significant contribution to the luminosity from recombination, and penetration of the photosphere into deep envelope layers, which did not initially (i.e., before the explosion) lie at a very small distance, $d r_{0} \ll R_{*}$, from the surface of the star. As long as the emission is dominated by shells with $d r_{0} / R_{*} \ll 1$, the luminosity and the color temperature are nearly independent of the pre-explosion density distribution. As the photosphere penetrates deeper, the emission becomes dependent on the details of the density distribution (see the "+" signs in Figures 2-4 of RW11, indicating the limit of model validity).
}

$10^{45} \mathrm{erg} \mathrm{s}^{-1}$ soft X-ray breakout signal of BSG explosions (which are expected to dominate the detection event rate) in archival searches of ROSAT (Vikhlinin 1998) and XMM (Law et al. 2004) data imply an upper limit of $\sim 10^{-7} \mathrm{Mpc}^{-3} \mathrm{yr}^{-1}$ on the rate of such events (Sapir et al. 2013; Sapir \& Halbertal 2014), which is about two orders of magnitude lower than the expected BSG explosion rate. This discrepancy may be related to the aforementioned tension between model predictions and observations, or to high obscuration of the explosions. In any case, it implies that a soft X-ray detector with a $1 \mathrm{sr}$ field of view (FOV) and sensitivity of $6 \times 10^{-11} \mathrm{erg} \mathrm{s}^{-1}$ (over $\sim 10 \mathrm{~s}$ ) will detect $<3$ events per year (consistent with the null detection of such events so far by MAXI; Camp et al. 2013). In a similar manner, we can estimate the detection rate of early $\mathrm{X}$-ray emission from $\mathrm{SNe}$ from the discovery of the early X-ray signal from SN 2008D by Soderberg et al. (2008). SWIFT-XRT could detect SN 2008D-like events out to $200 \mathrm{Mpc}$ (Soderberg et al. 2008). Even future wide-field Lobster telescopes will have a sensitivity that is $>100$ times less than XRT (Camp et al. 2013), and thus would detect such events only to $20 \mathrm{Mpc}$. This implies that even if the X-ray breakout rate is as high as the entire core-collapse SN rate, $\sim 10^{-4} \mathrm{Mpc}^{-3} \mathrm{yr}^{-1}$, such a future mission would detect three events per year (for an all sky detector).

iii. Finally, we note that massive star explosions associated with strong high-energy short transients, gamma-ray bursts (GRB), and X-ray flashes (XRF), like SN 2006aj (Campana et al. 2006), are both not understood theoretically and are very rare in the volumetric sense, as they account for $\ll 1 \%$ of core-collapse $\mathrm{SNe}$ (e.g., Podsiadlowski et al. 2004). The detection of such events cannot therefore be used to study the general properties of $\mathrm{SN}$ progenitor/explosion parameters.

Thus, there is strong motivation to study early SN emission in the UV. The bolometric luminosity of the early UV emission from $\mathrm{SNe}$ remains nearly constant, while the temperature of the cooling, expanding gas declines with time. In any given band, the measured flux will rise as the peak of the emitted spectrum cools and approaches the band center, reaching maximum when the spectral peak is within the band, then declining as further cooling drives the emission peak to redder wavelengths (Figure 2; see an animated version, Figure 3). The rate of cooling (and thus the time it takes for the flux to peak in a given band) depends on the stellar radius and the composition of the envelope, which determines the opacity. For supergiant explosions with thick hydrogen envelopes, the opacity is known (Thomson scattering) and time independent, so the radius is straightforwardly inferred (Figure 4). For evolved (e.g., W-R) stars, the opacity is a function of the surface composition (mass fraction of $\mathrm{He}, \mathrm{C}$, and $\mathrm{O}$ ). RW11 show that, given a well-sampled UV light curve, one can infer the stellar radius and constrain the surface composition (Figure 5).

Model calculations such as those of RW11 assumed standard massive star models, which leads to the prediction of a nearly constant shock-cooling luminosity. However, some recent works hint that at least some stars undergo violent preexplosion evolution (e.g., eruptive mass loss; Pastorello et al. 2007; Ofek et al. 2013, 2014a; Gal-Yam et al. 2014) and thus their pre-explosion density distribution may strongly 

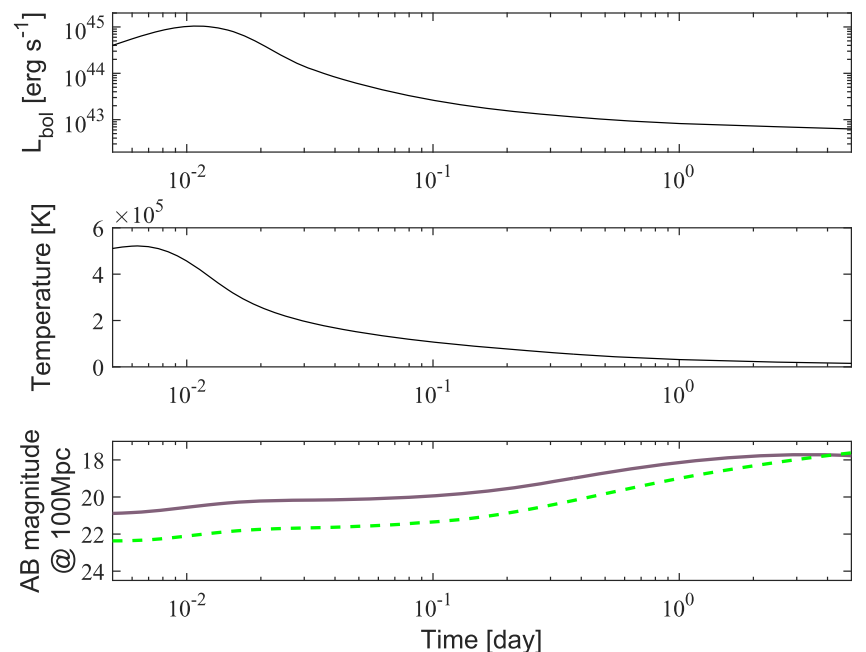

Figure 2. The bolometric luminosity (top), temperature (middle), and NUV (purple) and $g$-band (green) luminosities (bottom) predicted by the models of RW11 and SKW13 for a fiducial RSG SN progenitor with a radius of $500 R_{\odot}$, explosion energy of $1 \times 10^{51} \mathrm{erg}$, and ejected mass of $10 M_{\odot}$. The rapid decline of the temperature leads to an NUV peak around two days after explosion, when the blackbody peak temperature crosses this band, while the optical $g$-band continues to rise beyond day five. See Figure 3 for an animated version of this figure.

deviate from standard models. In this case, the shock-cooling luminosity will not be constant.

However, RW11 also show that by combining UV and optical data one can determine the exact extinction toward an event and, correcting for it, measure both the temperature evolution and the radius without any assumptions regarding a constant shock-cooling luminosity. For such events, the luminosity and temperature evolution extracted from the UV +optical data then measures the non-standard density profile, mapping recent pre-explosion mass loss and the physics of the final stages of stellar evolution.

In all cases, the extinction-corrected absolute luminosity evolution can be used to derive the energy per unit mass in the exploding ejecta $(E / M)$, which is yet another vital constraint on the explosion (Figure 6). The full route from UV light curves to physical stellar parameters has been demonstrated for (the only) three SN events with useful data (Types II and Ib; Gezari et al. 2008; Schawinski et al. 2008; Soderberg et al. 2008) by RW11.

Figure 7 shows how the progenitor radius and the explosion energy per unit mass can be directly derived from measurements of the UV peak absolute magnitude and rise time. Useful formulae to connect the observed parameters to the RW11 model parameters are provided below for radii $R_{*}$ measured in units of the solar radius $R_{\odot}$, energy $E$ in units of $10^{51} \mathrm{erg}$, and normalized to ejecta masses of $10 M_{\odot}$. We provide formulae for the absolute magnitude in the ULTRASAT band $\left(M_{\text {peak }}^{\text {USAT }}\right)$, as well as for the Swift UVW1 and UVW2 bands $\left(M_{\text {peak }}^{\mathrm{UVW} 1}, M_{\text {peak }}^{\mathrm{UVW} 2}\right)$. The rise time $t_{\text {rise }}$ is defined as the time in days it takes the UV magnitude to rise by 1 mag to peak.

$$
\begin{gathered}
M_{\text {peak }}^{\mathrm{USAT}}=-11.213-2.278 \log _{10}\left(R_{*}\right)-2.276 \log _{10}(E) \\
\log _{10}\left(t_{\text {rise }}\right)=-0.926+0.557 \log _{10}\left(R_{*}\right)+0.060 \log _{10}(E) \\
M_{\text {peak }}^{\mathrm{UVW} 1}=-11.221-2.278 \log _{10}\left(R_{*}\right)-2.276 \log _{10}(E)
\end{gathered}
$$
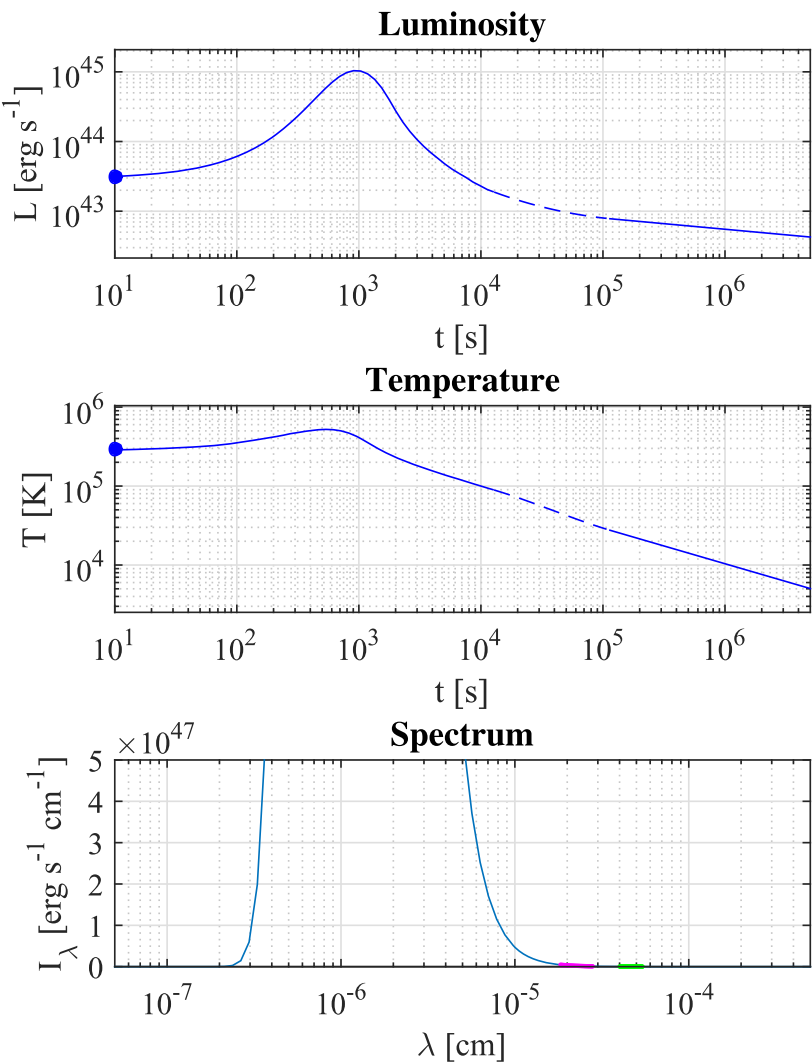

Light curves

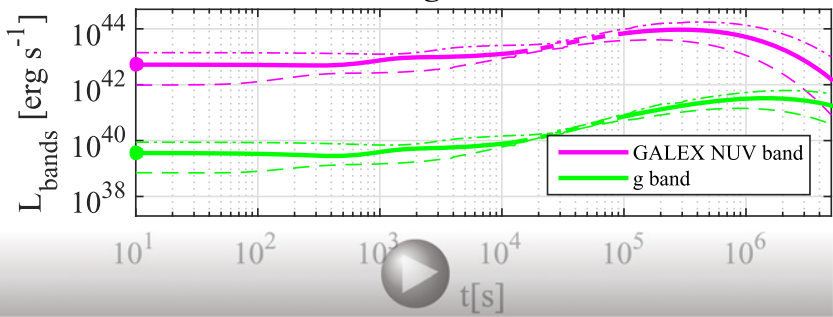

Figure 3. The evolution of the instantaneous spectrum and the derived luminosity in different bands. The bolometric luminosity (top), temperature (upper middle), spectrum (lower middle), and NUV (purple) and $g$-band (green) luminosities (bottom) predicted by the models of SKW13 and RW11 for a fiducial RSG SN progenitor with a radius of $500 R_{\odot}$, explosion energy of $1 \times 10^{51} \mathrm{erg}$, and ejected mass of $10 M_{\odot}$. The animation plots a full dot propagating in time along the bolometric luminosity, temperature, and band luminosity, as well as the instantaneous spectrum for that time. It uses a modified blackbody spectrum to describe the spectrum for the SKW13, rather than Wien one described in the paper because the comptonized spectrum peak is 10 times hotter than the bands' energy. The time axis for the SKW13 was arbitrarily shifted by $3 \times t_{0} \mathrm{~s}$ to include the rise to the peak luminosity and temperature in the graphs, which happens in negative times for this model (see explanation in the caption of Figure 1). The solid lines stand for exact solutions of the two models, while the dashed one represents an interpolation between them. The dashed thin lines in the bottom figure stand for $R=200 R_{\odot}$, while the dash dot thin lines stand for $R=1000 R_{\odot}$, both with the same explosion energies and mass.

(An animation of this figure is available.)

$\log _{10}\left(t_{\text {rise }}\right)=-0.913+0.556 \log _{10}\left(R_{*}\right)+0.060 \log _{10}(E)$

$M_{\text {peak }}^{\mathrm{UVW}}=-11.101-2.278 \log _{10}\left(R_{*}\right)-2.276 \log _{10}(E)$ 


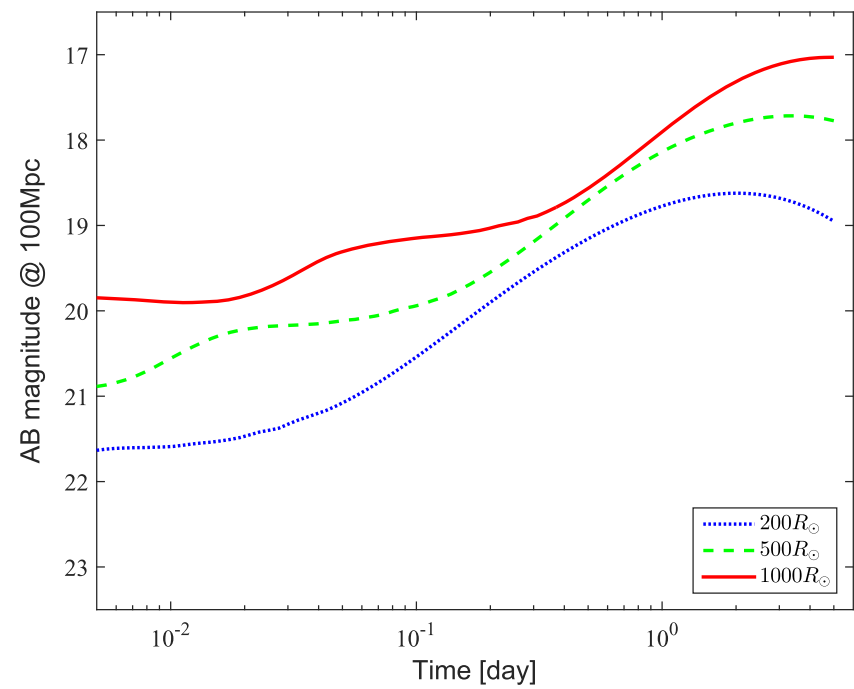

Figure 4. RW11 and SKW13 Model SN NUV light curves for RSG explosions with identical parameters except for the radius $R$ (explosion energy $E=1 \times 10^{51} \mathrm{erg}$, ejecta mass $M=10 M_{\odot}$ ). As can be seen, progenitor radii within the typical range for RSG stars $\left(200-1000 R_{\odot}\right)$ can be readily distinguished by the light curve shape (time to peak). Note that this diagnostic is independent of the absolute scale and so insensitive to extinction.

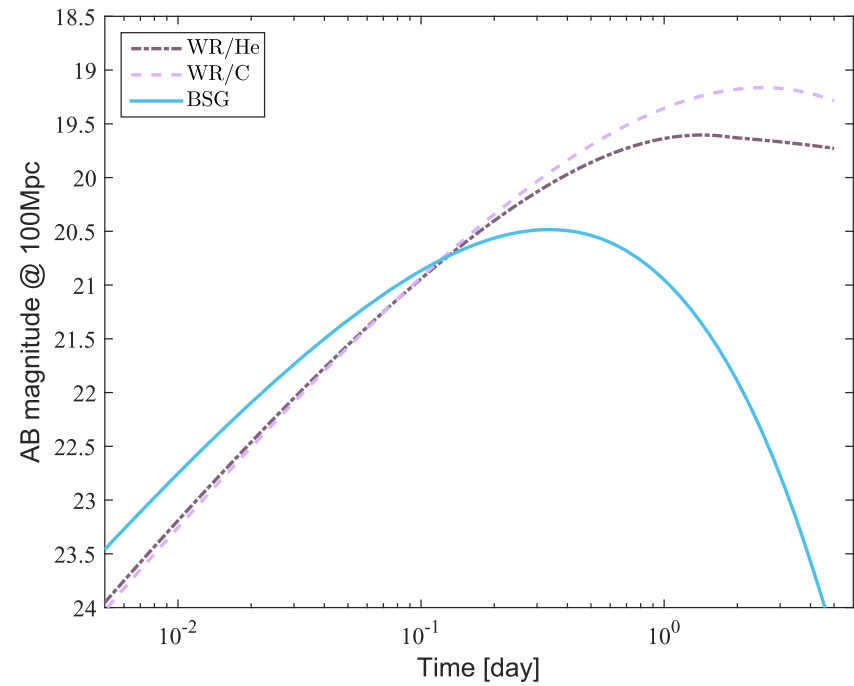

Figure 5. RW11 Model SN NUV light curves for explosions of BSG (hydrogen envelope; cyan) and $\mathrm{W}-\mathrm{R}$ (He and $\mathrm{C} / \mathrm{O}$ envelopes; light and dark purple, respectively) progenitors with identical radii $\left(10 R_{\odot}\right)$, explosion energy $\left(1 \times 10^{51} \mathrm{erg}\right)$, and mass $\left(5 M_{\odot}\right)$. Well-sampled early UV data $(<1$ day $)$ can readily diagnose both the radius and composition of compact stars. (The SKW13 model validity for these cases is earlier than the beginning of this graph time axis.)

$\log _{10}\left(t_{\text {rise }}\right)=-1.092+0.555 \log _{10}\left(R_{*}\right)+0.060 \log _{10}(E)$.

Early UV emission is thus a powerful way to study the progenitor properties of $\mathrm{SNe}$, motivating efforts to measure it systematically for a large sample of SN events by wide-field UV surveys. We now provide estimates of the expected SN detection rates by such surveys using observations and theory.

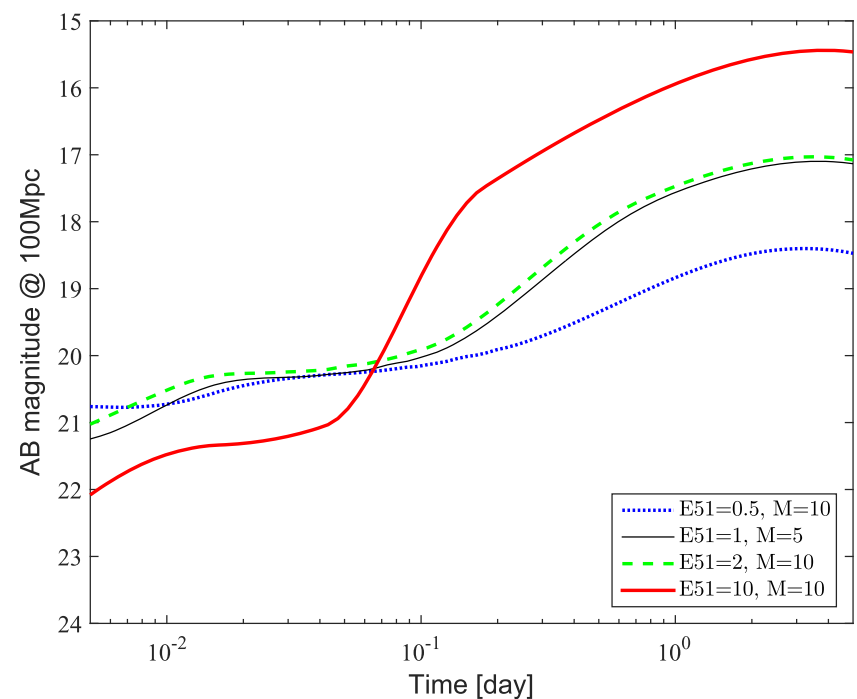

Figure 6. RW11 and SKW13 model SN NUV light curves for RSG stars with identical radii $\left(500 R_{\odot}\right.$ ) and several values of the explosion energy $E$ (in units of $E 51=10^{51} \mathrm{erg}$ ) and ejected mass $M$ (in solar mass). The RW11 ( $\gtrsim 0.3$ day) light curve shapes are identical (as these depend only on the radii and composition), while the luminosity is a function of the ratio $E / M$ (e.g., compare the dashed green and thin black curves). Assuming the extinction toward an event has been measured via the combination of UV and optical observations (RW11), one can use the luminosity to measure the value of $E / M$. Additional optical observations over longer timescales can constrain the ejected mass and allow us to independently infer both the explosion energy and the ejected mass separately (e.g., Barbarino et al. 2015).

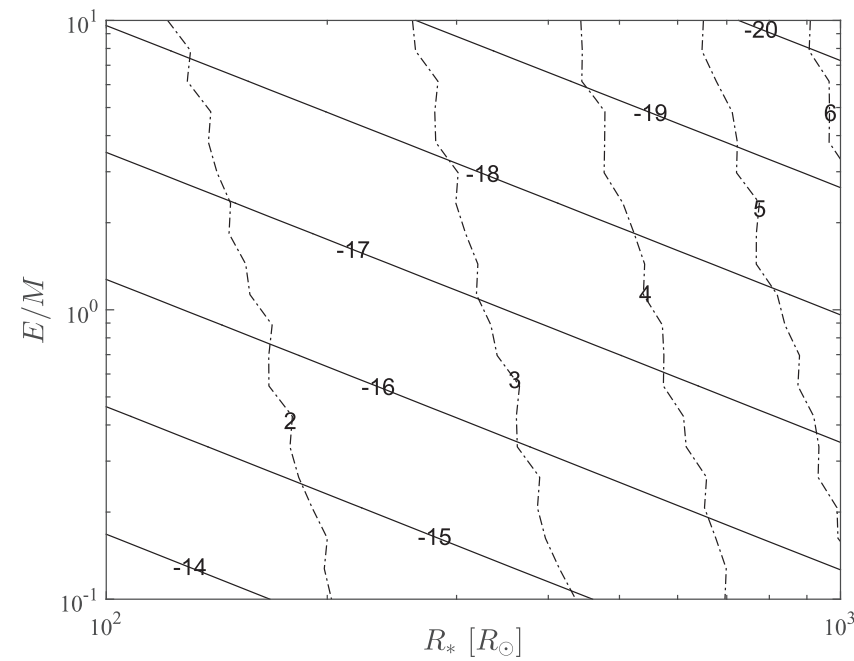

Figure 7. Lines of constant peak absolute UV magnitude in the ULTRASAT band (full line) and rise time in days (dash dot) predicted by RW11 models for RSG explosions as a function of the stellar radius $R$ and explosion mass $E / M$. As can be seen, the near-orthogonality of these lines allows us to simply deduce the underlying parameters from the observed peak and rise-time values.

\section{THE GALEX/PTF WIDE-FIELD, SHALLOW UV VARIABILITY SURVEY}

\subsection{Survey Description}

We conducted a UV wide-field transient survey during a nine-week period from 2012 May 24 to 2012 July 28. This survey used the GALEX NUV camera to cover a total area of 600 square degrees. Operating in scanning mode, the GALEX NUV camera observed strips of sky in a drift-scan mode with an effective average integration time of $80 \mathrm{~s}$, reaching a NUV 
Table 1

Sample of Core-collapse SNe Detected by the GALEX/PTF Experiment

\begin{tabular}{|c|c|c|c|c|c|}
\hline PTF Name & R.A. & Decl. & Redshift & Type & PTF Discovery Date \\
\hline PTF12ffs & $14: 42: 07.33$ & $+09: 20: 29.8$ & 0.0511 & $\mathrm{SN} \mathrm{II}^{\mathrm{a}}$ & 2012 Jun 10 \\
\hline PTF12fhz & $15: 18: 20.09$ & $+10: 56: 42.7$ & 0.0987 & SN IIb & 2012 Jun 12 \\
\hline PTF12fkp & $14: 46: 54.81$ & $+10: 31: 26.4$ & 0.12 & SN II-L & 2012 Jun 14 \\
\hline PTF12ftc & $15: 05: 01.88$ & $+20: 05: 54.6$ & 0.0732 & SN II-P & 2012 Jun 19 \\
\hline PTF12glz & $15: 54: 53.04$ & $+03: 32: 07.5$ & 0.0799 & SN IIn & 2012 Jul 7 \\
\hline PTF12gnt & $17: 27: 47.30$ & $+26: 51: 22.1$ & 0.029 & SN II-P & 2012 Jul 9 \\
\hline PTF12fes & $16: 00: 35.13$ & $+15: 41: 03.5$ & 0.0359 & SN Ib & 2012 Jun 9 \\
\hline PTF12fip & 15:00:51.04 & $+09: 20: 25.1$ & 0.034 & SN II-P & 2012 Jun 12 \\
\hline PTF12frn & $16: 22: 00.16$ & $+32: 09: 38.9$ & 0.136 & SN IIn & 2012 Jun 18 \\
\hline PTF12gcx & $15: 44: 17.32$ & $+09: 57: 43.1$ & 0.045 & $\mathrm{SN}$ II $^{\mathrm{b}}$ & 2012 Jun 26 \\
\hline
\end{tabular}

Notes.

a A bright SN II with a light curve intermediate between SNe II-P and II-L.

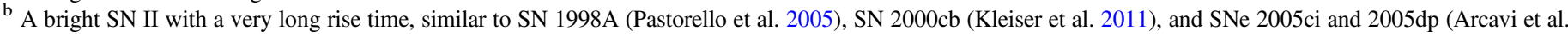
2012). See also Taddia et al. (2015).

limiting magnitude of 20.6 mag AB. Each strip was visited once every three days. In parallel, we observed the same area with the Palomar Transient Factory (PTF; Law et al. 2009; Rau et al. 2009) in the $r$-band, reaching a limiting magnitude of $R \sim 21$ mag $\mathrm{AB}$ with a cadence of two days, weather permitting.

The main scientific goals of this survey were to study the early UV emission from SNe (this work; N. Ganot et al. 2016, in preparation), AGN variability, stellar activity (flares), and white dwarf variability. We estimate the completeness of this survey to $\mathrm{SNe}$ exploding in the FOV and above the limiting magnitude (at some time) at $50 \%$, mainly due to the combined effects of GALEX data loss due to failed downlink and image corruption (about 20\%) and PTF weather losses and PTF survey incompleteness (60\% compounded). A full description of this survey, its completeness, and its results will be presented in a series of forthcoming papers.

\subsection{Detected SN Sample}

In this initial work we limit our analysis to the sample of spectroscopically confirmed SNe detected with PTF within the GALEX FOV during the survey period. The $\mathrm{SN}$ sample includes 33 Type Ia SNe that will be presented elsewhere, as well as 10 core-collapse $\mathrm{SNe}$. We list these core-collapse SNe in Table 1, and review their properties below. Interestingly, our survey also detected a distant superluminous SN of Type II (SLSN-II; Gal-Yam 2012) at $z=0.275$. This remarkable event (PTF12gwu; Figure 9) will be the subject of a separate publication. All of these events were spectroscopically classified as part of PTF operations and redshifts were measured from host galaxy lines (except for a single case, PTF12fkp, where the redshift is determined at lower accuracy from the SN lines).

We show the GALEX NUV light curves of the 10 corecollapse $\mathrm{SNe}$ in Figure 8. GALEX UV photometry was measured at the PTF SN locations using custom aperature photometry routines (Ofek 2014). We used an aperture of 5 pixels (7!"5). The sky was measured in an annulus with inner and outer radii of 20 and 50 pixels, and we used a zero point of $20.08 \mathrm{mag}$ and an aperture correction of $0.18 \mathrm{mag}$ for the GALEX NUV camera (Morrissey et al. 2007). The photometry is marked by solid circles with $1 \sigma$ error bars. PTF discovery dates are marked with dashed vertical lines. The vertical dot dashed line indicates when the optical light curve reaches $95 \%$ of its maximal value. Blue dotted horizontal lines indicate the flux level measured at these locations in pre-explosion GALEX data obtained prior to the start of our experiment. When such past imaging is not in hand, we indicate with dashed horizontal lines the quiescent flux level as measured from our GALEX data (the $25 \%$ percentile flux level, to avoid contamination by the $\mathrm{SN}$ flare emission). To assess detection significance, we calculated the $\chi^{2}$ and number of degrees of freedom obtained when fitting the data with a constant flux level, noted below each object name in Figure 8, where we also report in parenthesis the resulting false positive probability (FPA). Six events show clear UV flares (top panels; low FPA). Only four objects show no significant UV flare emission (bottom panels). Of those four events, two (PTF12fip and PTF12gcx) are consistent with a constant flux (solid gray line). Two other events (PTF12fes and PTF12frn) are inconsistent with a constant flux (low FPA) but show no clear flare-like structure. We conclude that six GALEX events are robustly detected.

Of those six, PTF photometry and spectroscopy indicates that they all are SNe II (2 SNe II-P, 1 intermediate II-P/L, 1 II$\mathrm{L}, 1 \mathrm{IIn}$ and $1 \mathrm{IIb}$; Table 1). The mean redshift of the GALEXdetected sample, as well as of the entire set of core-collapse $\mathrm{SNe}$ is $z \sim 0.07$. Interestingly, of the six GALEX-detected SNe, only one occurred in a luminous host, while four are located in dwarf galaxies and only marginally detected in our GALEX NUV observations. This indicates that for NUV-detected corecollapse $\mathrm{SNe}$, the host galaxy light contribution to the background is typically negligible. Most events are detected only during a small number of GALEX epochs (1-3) around their PTF discovery date, while the single detected Type IIn SN shows a prolonged period of UV luminosity extending beyond the duration of our survey period. Interestingly, in all cases the first UV detections occur prior to the optical discovery by PTF, elucidating the superiority of the UV over the optical for early SN studies.

We note that one out of these six events (PTF12glz) is a luminous Type IIn SN and displays a prolonged UV emission. The light curves of such events were suggested to be powered, at early times, via the explosion shock breaking out from a spatially extended opaque wind, rather than from the surface of a star (Ofek et al. 2010) and the decaying part is presumably due to the conversion of kinetic energy to optical luminosity 


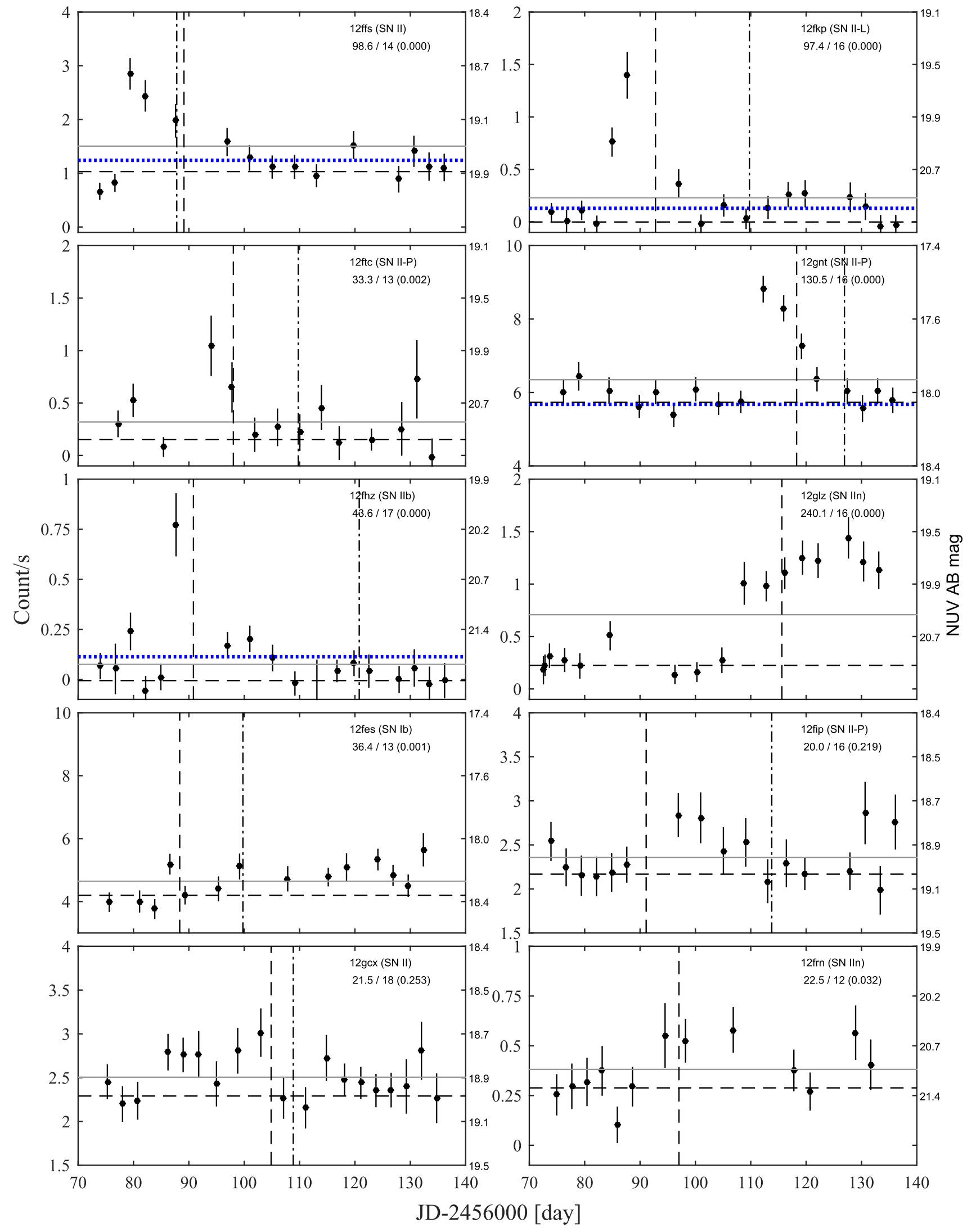

Figure 8. GALEX light curves of the 10 PTF spectroscopically confirmed core-collapse SNe (solid circles). PTF discovery dates are marked with dashed vertical lines. Vertical dot dashed line indicates when the optical light curve reaches $95 \%$ of its maximal value. Blue dotted horizontal lines indicate the flux level measured at these locations in pre-explosion GALEX data obtained prior to the start of our experiment; dashed horizontal lines are the quiescent flux level indicated from our own GALEX data (see text). Below each object name we report the $\chi^{2}$ per degrees of freedom obtained when fitting the data with a constant flux level, and in parenthesis the false alarm probability (FAP). Values below FAP $=0.001$ are marked as zero. Six events show clear UV flares (top panels). Only four objects show no significant UV flare emission (bottom panels; see text). 


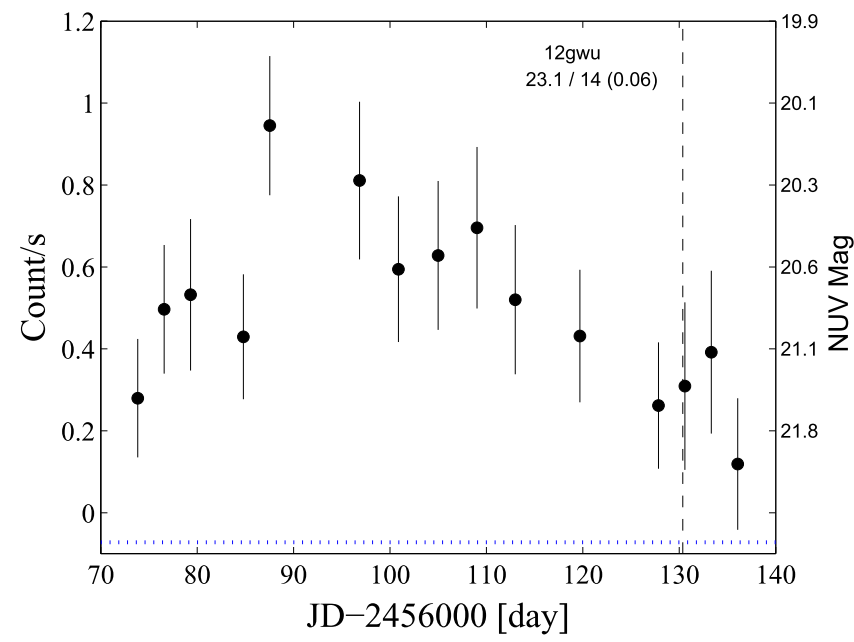

Figure 9. The GALEX light curve of PTF12gwu, a SLSN-II. Symbols and curves are the same as in Figure 8 . This event probably occurred around or shortly prior to the start of our GALEX/PTF experiment, which detected a very luminous and prolonged emission from this event. Analysis of these data will be presented in a forthcoming publication.

(see also Balberg \& Loeb 2011; Chevalier \& Irwin 2011, 2012; Moriya \& Tominaga 2012; Ginzburg \& Balberg 2014; Ofek et al. 2014b; Svirski \& Nakar 2014). Such events are relatively rare in the volumetric sense, but their detectability to larger distances compensates for this in flux-limited surveys. Our results indicate that in shallow UV surveys such events will constitute $15 \%-20 \%$ of the sample. An extreme such case are SLSNe, which are so UV-luminous that they are detected over a huge volume and may have similar detection rates (by number).

A brief report about these events was presented in Barlow et al. (2013), and a detailed analysis will be presented in N. Ganot et al. (2016, in preparation) and additional future publications.

\section{THEORETICAL ESTIMATES OF EARLY UV EMISSION FROM SNe}

\subsection{Light Curve Models and Comparison with Data}

We calculate theoretical early UV light curves for SNe in the following manner. We use the analytic formalism RW11, which has been tested against numerical simulations and selfsimilar solutions and describes available observations well (Section 2). Other analytical models (NS10; Chevalier 1992) are broadly similar and using those instead does not alter our derived detection rates. We are careful to correct the typographical error appearing in the RW11 formulae according to the published Erratum (Rabinak \& Waxman 2013).

Our calculations include the following steps. First, we calculate the RW11 bolometric luminosity for a set of progenitor parameters (radius $R_{*}$, explosion energy $E$, and ejected mass $M$ ). The model parameter $f_{\rho}$ is set to its suggested value of $f_{\rho}=0.1$. We use the Thomson opacity for supergiant stars, and the prescriptions of RW11 for mixed $\mathrm{He} / \mathrm{C} / \mathrm{O}$ envelopes of $\mathrm{W}-\mathrm{R}$ stars. We corrected the temperature up by a factor of 1.2, as suggested by RW11, to account for the fact that the color temperature is set at Thomson optical depth above unity (see NS10 for an analytic approximation). Using the evolving radius and temperature, we then calculate blackbody spectral curves as a function of time. Convolving these spectra with a sensitivity curve for a given observational band (e.g.,

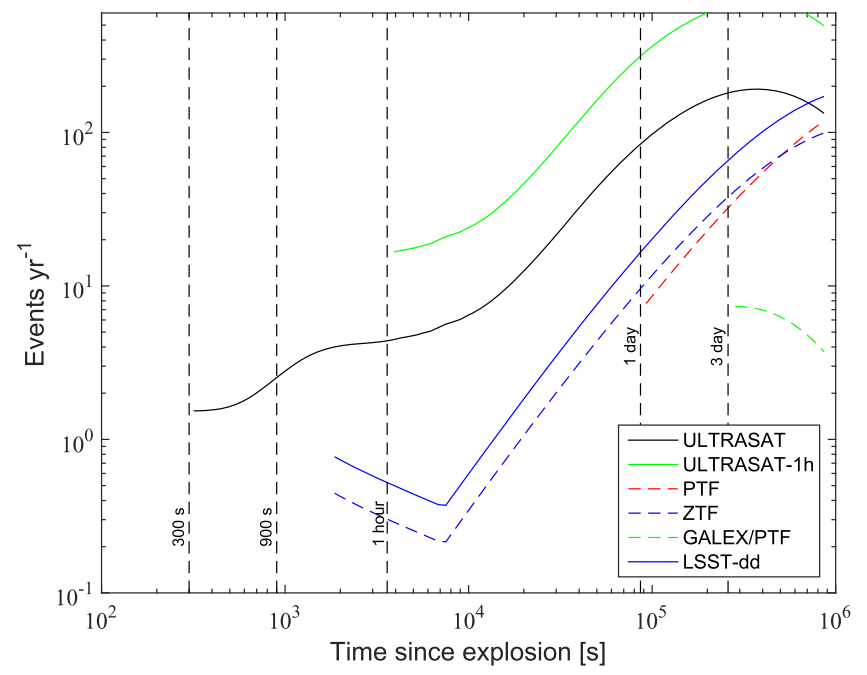

Figure 10. Expected number of early UV SN detections for various surveys. We use the models of SKW13 at early times and RW11 with our fiducial parameters $\left(R_{*}=500 R_{\odot}, E=10^{51} \mathrm{erg}\right.$, and $\left.M=10 M_{\odot}\right)$ at later times. The resulting expected detection rates for several surveys (Table 2) are plotted as a function of the time since explosion, starting with that expected for the survey minimal cadence. The curves are not cumulative (i.e., they indicate how many events will be detected at a given age, and not below that age). The survey parameters are given in Table 2.

NUV or optical bands), we calculate the light curve in these bands via synthetic photometry (Ofek 2014).

To determine object detectability, we assume a distance and a value for Galactic extinction, and calculate the flux from an object as a function of time since explosion. This is then translated to a number of detections for a survey with a given sensitivity (depth) in a given band and a given FOV (e.g., Figure 10), if we know the volumetric rate of the event in question.

\subsection{Volumetric Rates}

We use the volumetric SN rates from Li et al. (2011). Using a sample of nearby $\mathrm{SNe}$, these authors have measured rates for $\mathrm{SNe}$ II and Type $\mathrm{Ib} / \mathrm{c} \mathrm{SNe}$ of approximately $0.5 \times 10^{-4}$ $\mathrm{SN} \mathrm{Mpc}{ }^{-3} \mathrm{yr}^{-1}$ and $0.25 \times 10^{-4} \mathrm{SN} \mathrm{Mpc}^{-3} \mathrm{yr}^{-1}$, respectively. Since SNe II typically result from RSG stars (Smartt 2009), we normalize our predictions for RSG explosions using the SN II rate.

In contrast, the nature of the progenitors of $\mathrm{SNe} \mathrm{Ib} / \mathrm{c}$ is still unclear. While some indications exist that these arise from compact W-R stars (e.g., Corsi et al. 2012; Cao et al. 2013), other events appear to require more extended progenitors with radii well above $10^{11} \mathrm{~cm}$ (e.g., SN 2008D, RW11; SN 2006aj, Nakar \& Piro 2014). We have thus arbitrarily assigned $50 \%$ of the volumetric rate of $\mathrm{SNe} \mathrm{Ib} / \mathrm{c}$ to compact $\mathrm{W}-\mathrm{R}$ progenitors, and $50 \%$ to more extended BSG-like stars. For optical/UV surveys, the expected number of early detections of such compact stars are, in any case, small compared to the dominant population of RSG explosions.

\section{RESULTS}

Combining our UV light curve models and the measured SN volumetric rates, we can predict the expected number of detections of various progenitor explosions (RSG, BSG, and $\mathrm{W}-\mathrm{R}$ ) as a function of progenitor parameters $R_{*}, E$, and $M$. We begin by comparing our predictions with the $G A L E X / \mathrm{PTF}$ 
Table 2

Predicted SN Explosion Detection Numbers by Various Surveys

\begin{tabular}{|c|c|c|c|c|c|c|c|c|c|}
\hline \multirow[t]{2}{*}{ Survey } & \multirow[t]{2}{*}{ Band } & \multirow[t]{2}{*}{ Cadence } & \multirow[t]{2}{*}{ FOV $\left(\operatorname{deg}^{2}\right)$} & \multicolumn{6}{|c|}{ 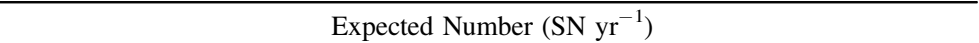 } \\
\hline & & & & $<1 \mathrm{hr}$ & $<1$ day & $<1 \mathrm{hr}$ & $<1$ day & $<1 \mathrm{hr}$ & $<1$ day \\
\hline ULTRASAT & NUV & $900 \mathrm{~s}$ & 210 & 4 & 85 & 1 & 8 & 0 & 3 \\
\hline ULTRASAT & NUV & $3600 \mathrm{~s}$ & 210 & 16 & 314 & 2 & 31 & 1 & 14 \\
\hline $\mathrm{ZTF}^{\mathrm{c}}$ & $g$ & $0.5 \mathrm{hr}$ & 2100 & 0 & 10 & 0 & 2 & 0 & 1 \\
\hline $\operatorname{LSST}^{\mathrm{d}}$ & $g$ & $0.5 \mathrm{hr}$ & 9.6 & 0 & 17 & 0 & 3 & 0 & 1 \\
\hline
\end{tabular}

Notes.

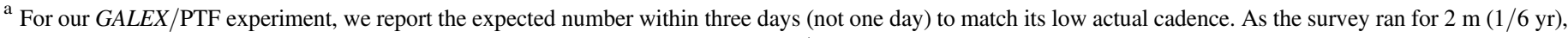
the expected number of SNe from RSG explosions for the actual experiment is $30 / 6=5$ events.

b Assumed temporal efficiency of $25 \%$ (including loss due to daytime and average weather) and lunation-averaged depth of 20.6 mag.

c $25 \%$ temporal efficiency as above, average depth $20.4 \mathrm{mag}$, and $50 \%$ survey time spent in $g$-band.

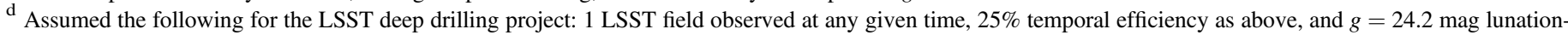
averaged depth.

survey we conducted (Section 5.1), and provide predictions for other space (Section 5.2) and ground-based (Section 5.3) surveys. We discuss the fractions of SN types in wide-field surveys in Section 5.4 based on observed PTF data.

\subsection{Predicted Rates for the GALEX/PTF Experiment and Fiducial Progenitor Parameters}

Using the procedure described previously, we predict the expected number of early UV detections of $\mathrm{SNe}$ II in our $G A L E X / \mathrm{PTF}$ experiment assuming all of these result from RSG progenitors with a single set of fiducial parameters. We set these to be $R_{*}=500 R_{\odot}, E=10^{51} \mathrm{erg}$, and $M=10 M_{\odot}$, which agree with typical values for RSG radii and energy and mass estimates for SNe II. Using this set of fiducial parameters and the RW11 models, we predict that our survey should have detected five $\mathrm{SNe}$ (Table 2; assuming it was 50\% complete, see above). Comparing this with the six actual detections (Section 3; Figure 8), we find good agreement with the predictions given the small numbers involved. We conclude that using the set of fiducial RSG model parameters and RW11 models to predict early UV SN detection numbers is validated by our GALEX/PTF experiment.

We calculate the number of BSG and W-R explosions using the parameters $R_{* \mathrm{BSG}}=50 R_{\odot}, E_{\mathrm{BSG}}=2 \times 10^{51} \mathrm{erg}$, and $M_{\mathrm{BSG}}=10 \quad M_{\odot} \quad$ and for $\mathrm{W}-\mathrm{R}$ stars $R_{* \mathrm{WR}}=10 \quad R_{\odot}$, $E_{\mathrm{WR}}=2 \times 10^{51} \mathrm{erg}$, and $M_{\mathrm{WR}}=10 M_{\odot}$, and the rates from Section 4.2. Even with these rather optimistic parameters (high $E$ and large $R$ ), the predicted number of BSG and $\mathrm{W}-\mathrm{R}$ explosion detections within the $G A L E X / \mathrm{PTF}$ experiment is small $(<1)$. This is consistent with our non-detection of early UV emission from SNe Ib/c (or the peculiar SN II PTF12gcx, which we suspect may have had a BSG progenitor; see Taddia et al. 2015). We retain these as fiducial parameters for predictions, but note they are not constrained by our observations.

\subsection{Predictions for ULTRASAT}

Having in hand a set of calibrated fiducial progenitor parameters for RSGs $\left(R_{*}=500 R_{\odot}, E=10^{51} \mathrm{erg}, M=10 M_{\odot}\right.$; RW11 models) that have been validated by reproducing our
GALEX observations, we can now predict the expected rates for the proposed ULTRASAT mission. For BSG and W-R explosions our parameters are poorly constrained by data, so any predictions are tentative, but the rate is expected to be dominated by RSG explosions (and it is). This wide-field UV space explorer has been described in Sagiv et al. (2014), and here we use its current technical formulation, a FOV of 210 square degrees and a $5 \sigma$ limiting sensitivity of 21.5 mag $\mathrm{AB}$ in $900 \mathrm{~s}$ integration in the NUV $(220-280 \mathrm{~nm}$ band $) .{ }^{19}$

As can be seen in Table 2, ULTRASAT is predicted to discover the early shock-cooling emission from no less than 96 events per year. Of these, the large majority (85) are expected to be due to RSG explosions. A handful of events (formally 4 per year) are expected to be detected during the shock-breakout phase ( $<1$ hour after explosion) but we only consider this number to be a tentative estimate because the theory of SN emission at this phase has not been tested observationally yet. We note that this prediction does not account for the extinction of these $\mathrm{SNe}$ in their hosts, but such an extinction will not affect the rate prediction. The reason is that we chose our fiducial RSG parameters to match the observed GALEX/PTF rate. If we include an arbitrary mean extinction in our modelling (reducing the expected number in the GALEX experiment), this would drive the RSG parameters toward values with brighter UV flares (larger $R$ or higher $E / M$ ) to exactly compensate and return the expected rate to its observed value. The effects of extinction thus cancel out and our predicted rates remain the same.

We can estimate the expected accuracy with which we can derive progenitor and explosion parameters from ULTRASAT data in the following manner. For our fiducial RW11 models, we calculate the covariance matrix taking $R_{*}, E / M$, and the explosion time $t_{0}$ as free parameters; Poisson errors appropriate for the distance of a given event; its expected luminosity; and the ULTRASAT sensitivity (limiting magnitude of $M_{\mathrm{NUV}}=21.5 \mathrm{AB}$ Mag during $900 \mathrm{~s}$ integrations), as well as $3 \%$ systematic errors. The square root of the diagonal elements of the covariance matrix are reported in Figure 11. As can be

\footnotetext{
${ }^{19}$ http://www.weizmann.ac.il/astrophysics/ultrasat/
} 


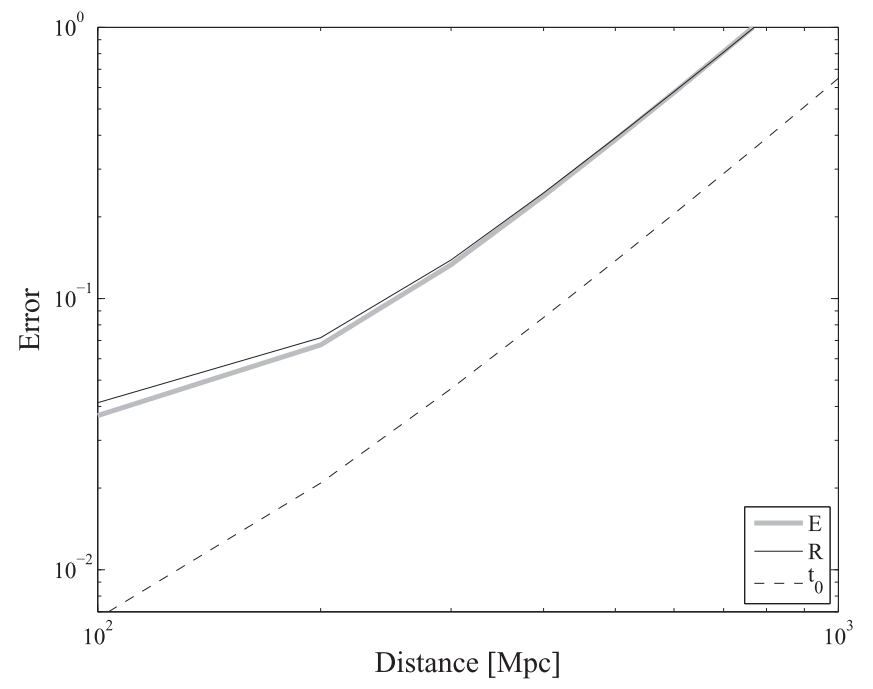

Figure 11. The expected fractional errors on supernova progenitor and explosion parameters derived from covariance matrix analysis for RW11 models with our fiducial RSG progenitor parameters; see text for details.

seen, ULTRASAT will provide accurate measurements of these parameters (to below $10 \%$ out to $200 \mathrm{Mpc}$ ).

Another simple, robust, and extinction-free lower limit on the ULTRASAT detection rate is obtained by scaling our GALEX results. Our GALEX experiment detected $N_{G A L E X}=6$ events in $\mathrm{t}=2$ months using a survey area of $\Omega=600 \mathrm{deg}^{2}$, which had a $5 \sigma$ flux sensitivity of $\mathrm{S}_{\mathrm{NUV}}=20.6 \mathrm{mag} \mathrm{AB}$ and covered a sky area with $\delta A_{\mathrm{NUV}}=0.31 \mathrm{mag}$ higher NUV extinction compared to the ULTRASAT NUV-optimized fields. Thus, direct scaling to the ULTRASAT yearly yield would give $N_{\text {ULTRASAT }}=\mathrm{N}_{\text {GALEX }} \times(1$ year $/ 2$ months $) \times$ $(210 / 600) \times\left(S_{\text {ULTRASAT }}-S_{\text {GALEX }}+\delta A_{\mathrm{NUV}}\right)^{3 / 2}, \quad$ which yields $N_{\text {ULTRASAT }}=71$ events $\mathrm{yr}^{-1}$. This lower limit is based purely on NUV-detected $\mathrm{SNe}$, so it accounts for all sources of extinction. It is also a conservative lower limit because it uses the rate from the $G A L E X / \mathrm{PTF}$ survey, which had a UV cadence of three days, compared to the expected ULTRASAT cadence of $900 \mathrm{~s}$. Many short events likely missed by our GALEX/PTF search could be detected by ULTRASAT. Finally, this assumes a $100 \%$ efficiency for our $G A L E X / \mathrm{PTF}$ experiment, while in reality its completeness was no more than $\sim 50 \%$ (see above). Correcting just for this factor, our lower limit is in good agreement with the theoretical prediction. The formal error on this lower limit is due to Poisson small-number statistics related to the GALEX detection number (six). At $95 \%$ confidence, this error is by a factor of two or less (Gehrels 1986).

We conclude that ULTRASAT is absolutely guaranteed to find $>85$ explosions of large RSG stars per year using this direct scaling from our GALEX observations, and that with high confidence this number is twice as large. The predicted detection rate $R$ for other UV missions operating in a similar wavelength range can be estimated using simple scaling according to FOV $\Omega$ and limiting flux $S, R \propto \Omega \times S^{3 / 2}$.

An interesting final point is that our $G A L E X / \mathrm{PTF}$ experiment discovered one superluminous SN (SLSN-II) in two months. Using the same scaling above for ULTRASAT indicates this mission will likely detect $\sim 10$ SLSNe per year. These will be unique in having been discovered early and having UV coverage, which is crucial to shed light on the progenitors and power sources of these most energetic and UVbright objects.

\subsection{Predictions for Other Surveys}

The same models we apply above can also be used to predict the expected early SN discovery rate for other experiments, in particular ground-based optical surveys. We note that these surveys would not be able to carry out the science investigation motivated in Section 2, because it requires early UV data. However, these ground-based surveys could conceivably trigger UV follow-up observations from space (e.g., by Swift or even Hubble Space Telescope) that will allow progenitor and $\mathrm{SN}$ physics to be extracted from early data, at least for a few objects.

The iPTF survey at Palomar Observatory is operating the PTF survey camera and has demonstrated its ability to quickly discover SNe and trigger space-based UV follow-up (Gal-Yam et al. 2011). We calculate the expected number of events for an iPTF survey covering $1000 \mathrm{deg}^{2}$ with a nightly cadence in the $r$-band (Table 2). iPTF has a lunation-averaged depth of $r=20.6 \mathrm{mag}$, and we assumed a $25 \%$ temporal efficiency (including loss due to daytime and weather). The predicted yearly yield (nine events) is consistent with iPTF detections of several SNe at ages $<1$ day so far (e.g., Gal-Yam et al. 2014).

Next, we consider the coming Zwicky Transient Facility (ZTF), which will be using an even larger camera mounted on the same telescope at Palomar Observatory. While the observing strategy of ZTF has not been finalized yet, we consider here a $g$-band survey of $2100 \mathrm{deg}^{2}$ with a cadence of $0.5 \mathrm{hr}$. We assume this survey will use $50 \%$ of the ZTF time, and the same temporal efficiency as above. With a dozen or so events per year, all securely detected at $<1$ day, ZTF, hopefully coupled with Swift, will be able to provide the first few examples of the science expected from ULTRASAT.

Finally, it is interesting to consider what the Large Synoptic Survey Telescope (LSST) could achieve. Assuming that, as part of a "deep drilling" experiment, LSST will observe a single FOV at any given time repeatedly every $0.5 \mathrm{hr}$ in the $g$ band with a lunation-averaged depth of $g=24.2 \mathrm{mag}$ and a temporal efficiency as above, LSST will be able to perhaps detect less than $1 \mathrm{SN}$ per year within $1 \mathrm{hr}$ of the explosion, and about 20 events per year within 1 day of the explosion. This is still well below the expected performance of ULTRASAT. In addition, these events will typically be distant, faint, and difficult to follow-up (e.g., spectroscopically).

\subsection{Estimated SN Fractions in Wide-field Surveys}

In view of the complexity of massive stars and the resulting diversity of their explosive core-collapse SN outcomes, an important aspect in the design of a survey to systematically study early emission from massive star explosions is the number of different SN types one expects to obtain significant data about. To assess this for our own survey, as well as future programs such as ULTRASAT (Sagiv et al. 2014), we use the large sample of spectroscopically confirmed CC SNe from the PTF survey (Law et al. 2009; Rau et al. 2009; Arcavi et al. 2010). The final sample from PTF (2009-2012) includes 484 events. This sample is suitable for our study because it comes from a relatively shallow survey with a depth identical to the $G A L E X / \mathrm{PTF}$ survey (by definition) and similar to that predicted for ULTRASAT. The survey is also untargeted (it 
Table 3

SN Fractions from 484 PTF Core-collapse SNe and Predictions from Other Surveys

\begin{tabular}{|c|c|c|c|c|c|c|}
\hline SN Type & PTF Number & $\begin{array}{c}\text { Fraction } \\
\text { of Total }(\%)\end{array}$ & $\begin{array}{c}\text { Expected \# } \\
(\text { per 100) }\end{array}$ & $\begin{array}{l}\text { Minimum \# } \\
(95 \% \text { c.l. })^{\mathrm{a}}\end{array}$ & $\begin{array}{c}\text { Expected } \\
\text { (per 10) }\end{array}$ & $\begin{array}{c}\text { Actual } \\
\text { GALEX/PTF }\end{array}$ \\
\hline II-P & 193 & 40 & 40 & 30 & 4 & 4 \\
\hline II-L & 70 & 14 & 14 & 8 & 1 & 1 \\
\hline IIn & 91 & 19 & 19 & 12 & 2 & 2 \\
\hline II-pec ${ }^{\text {b }}$ & 5 & 1 & 1 & 0 & 0 & 1 \\
\hline $\mathrm{Ib}$ & 34 & 7 & 7 & 3 & 1 & 1 \\
\hline
\end{tabular}

Notes.

${ }^{a}$ Minimum number expected per 100 events at a confidence level of 95\% calculated using small-number Poisson statistics (Gehrels 1986).

${ }^{\mathrm{b}}$ Peculiar SNe II with very long rise times, similar to SN 1987A or PTF12gcx (see note to Table 1).

is not focused on known cataloged galaxies that are typically biased toward more massive and metal-rich objects).

The fractions of SNe of different types from the PTF fluxlimited sample are reported in Table 3. The separation of the common class of SNe II into photometric subclasses (II-P and II-L) should not be regarded as final, and is, in any case, controversial (e.g., Arcavi et al. 2012; Anderson et al. 2014; Faran et al. 2014a, 2014b). The size of the PTF sample allows estimates of the observed fractions of even rare classes (e.g., IcBL and II-pec) with reasonable accuracy.

Table 3 also provide estimates for a fiducial sample of 100 events, as well as the $95 \%$ confidence lower limit on the expected number of $\mathrm{SNe}$ from each class in this fiducial sample. We find that all SN types except for the rare Type IIpec events are expected to be detected in samples of 100 events or larger.

Next, we estimate the expected yield of our $G A L E X / \mathrm{PTF}$ experiment and compare it with our actual findings (right columns of Table 3). We find excellent agreement even for the small numbers in question. We counted PTF12ffs as a Type II$P$ event, but including it in the II-L class would not significantly alter this result in view of the small SN numbers.

\section{CONCLUSIONS}

Motivated by the scientific promise of early UV observations of $\mathrm{SNe}$, we conducted a GALEX/PTF survey for such events that detected six $\mathrm{SNe}$ II at ages $<3$ day. We develop a theoretical framework to predict the number of early UV SN detections in general surveys using theoretical UV light curves that fit existing data well, combined with measured volumetric $\mathrm{SN}$ rates. We find that adopting a set of reasonable physical parameters for exploding RSG SN progenitors $\left(R_{*}=500 R_{\odot}\right.$, $E=10^{51} \mathrm{erg}$ and $\left.M=10 M_{\odot}\right)$ fits our PTF $/ G A L E X$ results well. We adopt these parameters and predict the expected early UV SN detection numbers from the proposed ULTRASAT space mission, as well as several ground-based surveys (Table 2). We find that ULTRASAT is expected to discover $>85 \mathrm{SNe}$ per year in the UV, within 1 day of explosion. A robust lower limit directly derived from the GALEX UV detection rates supports this estimate. Using SN Type statistics from PTF, we show that such a sample is likely to include examples of all common SN Types (Table 3). We conclude that a space mission like ULTRASAT will be able to comprehensively map the progenitor properties of $\mathrm{SNe}$ of all types (including radii and surface composition) and constrain SN explosion physics, providing a compelling answer to the question of massive stellar death.

This research was supported by grants from the Israeli Space Agency (ISA) and the Ministry of Science, Technology and Space (MOS). Additional funding was provided by the EU via ERC grant 307260, the ISF, and a Kimmel award.

\section{REFERENCES}

Anderson, J. P., González-Gaitán, S., Hamuy, M., et al. 2014, ApJ, 786, 67 Arcavi, I., Gal-Yam, A., Cenko, S. B., et al. 2012, ApJL, 756, L30 Arcavi, I., Gal-Yam, A., Kasliwal, M. M., et al. 2010, ApJ, 721, 777 Arcavi, I., Gal-Yam, A., Yaron, O., et al. 2011, ApJL, 742, L18 Arnett, W. D., Bahcall, J. N., Kirshner, R. P., \& Woosley, S. E. 1989, ARA\&A, 27, 629

Balberg, S., \& Loeb, A. 2011, MNRAS, 414, 1715

Barbarino, C., Dall'Ora, M., Botticella, M. T., et al. 2015, MNRAS, 448, 2312

Barlow, T. A., Gal-Yam, A., \& Ofek, E. 2013, BAAS, 221, 215.10

Camp, J., Barthelmy, S., Blackburn, L., et al. 2013, ExA, 36, 505

Campana, S., Mangano, V., Blustin, A. J., et al. 2006, Natur, 442, 1008

Cao, Y., Kasliwal, M. M., Arcavi, I., et al. 2013, ApJL, 775, L7

Chevalier, R. A. 1976, ApJ, 207, 872

Chevalier, R. A. 1992, ApJ, 394, 599

Chevalier, R. A., \& Fransson, C. 2008, ApJL, 683, L135

Chevalier, R. A., \& Irwin, C. M. 2011, ApJL, 729, L6

Chevalier, R. A., \& Irwin, C. M. 2012, ApJL, 747, L17

Colgate, S. A. 1974, ApJ, 187, 333

Corsi, A., Ofek, E. O., Gal-Yam, A., et al. 2012, ApJL, 747, L5

Ensman, L., \& Burrows, A. 1992, ApJ, 393, 742

Falk, S. W. 1978, ApJL, 225, L133

Faran, T., Poznanski, D., Filippenko, A. V., et al. 2014a, MNRAS, 445, 554

Faran, T., Poznanski, D., Filippenko, A. V., et al. 2014b, MNRAS, 442, 844 Filippenko, A. V. 1997, ARA\&A, 35, 309

Gal-Yam, A. 2012, Sci, 337, 927

Gal-Yam, A., Arcavi, I., Ofek, E. O., et al. 2014, Natur, 509, 471

Gal-Yam, A., Kasliwal, M. M., Arcavi, I., et al. 2011, ApJ, 736, 159

Gal-Yam, A., Mazzali, P. A., Manulis, I., \& Bishop, D. 2013, PASP, 125, 749 Gehrels, N. 1986, ApJ, 303, 336

Gezari, S., Dessart, L., Basa, S., et al. 2008, ApJL, 683, L131

Gezari, S., Rest, A., Huber, M. E., et al. 2010, ApJL, 720, L77

Ginzburg, S., \& Balberg, S. 2014, ApJ, 780, 18

Grassberg, E. K., Imshennik, V. S., \& Nadyozhin, D. K. 1971, Ap\&SS, 10, 28

Katz, B., Sapir, N., \& Waxman, E. 2012, ApJ, 747, 147

Klein, R. I., \& Chevalier, R. A. 1978, ApJL, 223, L109

Kleiser, I. K. W., Poznanski, D., Kasen, D., et al. 2011, MNRAS, 415, 372

Langer, N. 2012, ARA\&A, 50, 107

Law, N. M., Kulkarni, S. R., Dekany, R. G., et al. 2009, PASP, 121, 1395

Law, N. M., Rutledge, R. E., \& Kulkarni, S. R. 2004, MNRAS, 350, 1079

Li, W., Chornock, R., Leaman, J., et al. 2011, MNRAS, 412, 1473

Matzner, C. D., \& McKee, C. F. 1999, ApJ, 510, 379

Moriya, T. J., Sanyal, D., \& Langer, N. 2015, A\&A, 575, L10

Moriya, T. J., \& Tominaga, N. 2012, ApJ, 747, 118

Morrissey, P., Conrow, T., Barlow, T. A., et al. 2007, ApJS, 173, 682 
Nakar, E., \& Piro, A. L. 2014, ApJ, 788, 193

Nakar, E., \& Sari, R. 2010, ApJ, 725, 904

Ofek, E. O. 2014, ascl soft, ascl:1407.005

Ofek, E. O., Arcavi, I., Tal, D., et al. 2014a, ApJ, 788, 154

Ofek, E. O., Rabinak, I., Neill, J. D., et al. 2010, ApJ, 724, 1396

Ofek, E. O., Sullivan, M., Cenko, S. B., et al. 2013, Natur, 494, 65

Ofek, E. O., Sullivan, M., Shaviv, N. J., et al. 2014b, ApJ, 789, 104

Pastorello, A., Baron, E., Branch, D., et al. 2005, MNRAS, 360, 950

Pastorello, A., Smartt, S. J., Mattila, S., et al. 2007, Natur, 447, 829

Podsiadlowski, P., Mazzali, P. A., Nomoto, K., Lazzati, D., \& Cappellaro, E. 2004, ApJL, 607, L17

Pritchard, T. A., Roming, P. W. A., Brown, P. J., Bayless, A. J., \& Frey, L. H. 2014, ApJ, 787, 157

Rabinak, I., \& Waxman, E. 2011, ApJ, 728, 63

Rabinak, I., \& Waxman, E. 2013, ApJ, 770, 81
Rau, A., Kulkarni, S. R., Law, N. M., et al. 2009, PASP, 121, 1334

Rubin, A., Gal-Yam, A., De Cia, A., et al. 2015, ApJ, in press (arXiv:1512.00733)

Sagiv, I., Gal-Yam, A., Ofek, E. O., et al. 2014, AJ, 147, 79

Sapir, N., \& Halbertal, D. 2014, ApJ, 796, 145

Sapir, N., Katz, B., \& Waxman, E. 2011, ApJ, 742, 36

Sapir, N., Katz, B., \& Waxman, E. 2013, ApJ, 774, 79

Schawinski, K., Justham, S., Wolf, C., et al. 2008, Sci, 321, 223

Schmidt, B. P., Kirshner, R. P., Eastman, R. G., et al. 1993, Natur, 364,600

Smartt, S. J. 2009, ARA\&A, 47, 63

Soderberg, A. M., Berger, E., Page, K. L., et al. 2008, Natur, 453, 469

Svirski, G., \& Nakar, E. 2014, ApJ, 788, 113

Taddia, F., Sollerman, J., Leloudas, G., et al. 2015, A\&A, 574, A60

Vikhlinin, A. 1998, ApJL, 505, L123 